\title{
Novel first-dose adverse drug reactions during a phase I trial of olipudase alfa (recombinant human acid sphingomyelinase) in adults with Niemann-Pick disease type B (acid sphingomyelinase deficiency)
}

\author{
Margaret M. McGovern, MD, PhD ${ }^{1,4}$, Melissa P. Wasserstein, MD¹, Brian Kirmse, MD1, W. Lane Duvall, MD', \\ Thomas Schiano, MD¹, Beth L. Thurberg, MD, PhD², Susan Richards, PhD² and Gerald F. Cox, MD, PhD ${ }^{2,3}$
}

Purpose: Enzyme replacement therapy with olipudase alfa (recombinant human acid sphingomyelinase) is being developed for Niemann-Pick disease type B (NPD B).

Methods: A single-center, open-label, nonrandomized, singleascending-dose trial evaluated the safety of intravenous olipudase alfa $(0.03-1.0 \mathrm{mg} / \mathrm{kg})$ in 11 adults with NPD B. Patients were monitored in the hospital for $72 \mathrm{~h}$ after infusion and had follow-up visits on days 14 and 28.

Results: Plasma ceramide, a product of sphingomyelin catabolism by olipudase alfa, showed dose-dependent elevations by $6 \mathrm{~h}$ postdose, or postinfusion. No serious adverse drug reactions (ADRs) occurred during the study. Acute phase reaction-type ADRs, as evidenced by elevated inflammatory biomarkers (high-sensitivity C-reactive protein, interleukin-8, and calcitonin) and constitutional symptoms (fever, pain, nausea, and/or vomiting) emerged
$12-24 \mathrm{~h}$ following doses $\geq 0.3 \mathrm{mg} / \mathrm{kg}$ olipudase alfa. Three patients experienced hyperbilirubinemia. The study was terminated after a patient dosed at $1 \mathrm{mg} / \mathrm{kg}$ exhibited severe hyperbilirubinemia; he was subsequently diagnosed with Gilbert syndrome.

Conclusion: The maximum tolerated dose of olipudase alfa in adults with NPD B was $0.6 \mathrm{mg} / \mathrm{kg}$. First-dose ADRs were likely induced by elevated concentrations of ceramide (or its downstream derivatives) generated by the catabolism of accumulated sphingomyelin. Within-patient dose escalation to slowly catabolize sphingomyelin stores may be a strategy to mitigate first-dose ADRs in patients with NPD B.

Genet Med advance online publication 2 April 2015

Key Words: acute phase reaction; ceramide; hyperbilirubinemia; Niemann-Pick disease type B; recombinant human acid sphingomyelinase

\section{INTRODUCTION}

Enzyme replacement therapy (ERT) is available for several lysosomal storage disorders in which an inherited deficiency of a degradative enzyme results in pathogenic buildup of its substrate. Regular intravenous infusions of recombinant enzyme can compensate for the deficiency, reversing or stabilizing somatic disease and improving quality of life. ${ }^{1}$ The most common adverse drug reactions (ADRs) that occur with ERT are infusion-associated reactions (IARs) characterized by hypersensitivity-type symptoms (e.g., urticaria, rash, fever, chills, bronchospasm) that emerge during or immediately following an infusion. IARs typically arise several weeks after treatment initiation following repeated exposure to the drug and subsequently diminish over time. In many patients the onset of IARs coincides with the detection of IgG (and, rarely, IgE) antibodies against the infused enzyme., ${ }^{2,3}$

ERT with olipudase alfa (recombinant human acid sphingomyelinase) is in clinical development for acid sphingomyelinase deficiency (ASMD), historically known as Niemann-Pick disease (NPD; OMIM 607616) type A (NPD A) and type B (NPD B). Sphingomyelin, a major plasma membrane phospholipid, is hydrolyzed by acid sphingomyelinase (ASM) (EC 3.1.4.12) in lysosomes to yield ceramide and phosphorylcholine. Deficient enzyme activity causes sphingomyelin to accumulate within many cell types, especially tissue macrophages and hepatocytes. ${ }^{4}$ NPD encompasses a broad spectrum of clinical phenotypes, ranging from a neuronopathic form with severe somatic disease, failure to thrive, and death during early childhood (NPD A) to a nonneuronopathic form with survival usually into adulthood and variable degrees of hepatosplenomegaly, cytopenias, interstitial lung disease, and dyslipidemia (NPD B). ${ }^{5-8}$ No definitive disease-modifying treatment is available for ASMD despite attempts to use splenectomy, pulmonary lavage, liver transplantation, and hematopoietic stem cell transplantation. ${ }^{9-13}$

Preclinical studies in an ASM knockout mouse model of NPD demonstrated that biweekly administration of olipudase alfa reduced tissue sphingomyelin concentrations in a dosedependent manner. ${ }^{14,15}$ No adverse effects were observed at

\footnotetext{
${ }^{1}$ Icahn School of Medicine at Mount Sinai, New York, New York, USA $;{ }^{2}$ Genzyme, a Sanofi Company, Cambridge, Massachusetts, USA; ${ }^{3}$ Boston Children's Hospital and Harvard Medical School, Boston, Massachusetts, USA; ${ }^{4}$ Present address: Stony Brook University School of Medicine, Stony Brook, New York, USA. Correspondence: Gerald F. Cox (gerald.cox@genzyme.com)

Submitted 25 November 2014; accepted 21 January 2015; advance online publication 2 April 2015. doi:10.1038/gim.2015.24
} 
single doses as high as $0.3 \mathrm{mg} / \mathrm{kg}$, but doses $\geq 10 \mathrm{mg} / \mathrm{kg}$ caused liver inflammation and degeneration, adrenal hemorrhage, cardiovascular shock, and death..$^{14,15}$ Marked elevations in interleukin (IL)-6, granulocyte colony-stimulating factor, and keratinocyte-derived cytokine were detected within hours of dosing, consistent with cytokine storm..$^{15}$ This unexpected toxicity occurred after a single dose of olipudase alfa, precluding the involvement of antibody-mediated pathways. Normal animals did not exhibit a similar response, suggesting that the toxicity in ASM knockout mice was related to rapid elevations in the end product(s) of sphingomyelin hydrolysis. Additional studies demonstrated that the highdose toxicity could be completely prevented by prior treatment with several low doses to gradually debulk accumulated sphingomyelin. ${ }^{15}$

Herein we report results from the first-in-human study of olipudase alfa. This single-ascending-dose trial was designed to evaluate the dose-limiting toxicity (DLT) of olipudase alfa in adults with NPD B. The study incorporated precautionary measures to maximize patient safety, including the administration of olipudase alfa in a cardiac care unit during a 3-day inpatient stay, frequent assessments of vital organ function through 14 days after dosing, sequential infusion of patients, and analysis of all dose cohort data before dose escalation.

\section{MATERIALS AND METHODS}

\section{Study design}

This single-center, open-label, nonrandomized, phase I (NCT00410566) trial ran from December 2006 to March 2009. The institutional review board at Mount Sinai School of Medicine approved the protocol. Patients provided written consent before screening. Each study patient was infused with one dose of olipudase alfa $(0.03,0.1,0.3,0.6$, or $1.0 \mathrm{mg} / \mathrm{kg})$. The lowest dose corresponded to one-tenth of the single-dose, noobserved-adverse-effect level in ASM knockout mice. Patients were infused at $\geq 24$-h intervals within a dose cohort and $\geq 4$ weeks between dose cohorts. An independent data monitoring committee reviewed all safety data and made recommendations regarding further dosing if any of the following criteria for a potential DLT were met: $>3$-fold increase in aspartate aminotransferase (AST), alanine transaminase (ALT), alkaline phosphatase, or bilirubin; >1.5-fold increase in bilirubin or alkaline phosphatase accompanied by abovenormal AST or ALT; >10-fold increase in cytokines; a serious adverse event (AE); or any AE that raised a safety concern.

\section{Patient eligibility}

Inclusion criteria were 18-65 years of age, confirmed deficiency of ASM enzyme activity, spleen volume more than or equal to two multiples of normal (based on a normal spleen volume of $0.2 \%$ of body weight), diffusing capacity of the lung for carbon monoxide $>30 \%$ predicted, AST and ALT $\leq 250 \mathrm{IU} / \mathrm{l}$, bilirubin $\leq 61.6 \mu \mathrm{mol} / \mathrm{l}$, international normalized ratio $\leq 1.5$, and platelet count $\geq 60 \times 10^{9} / 1$. Exclusion criteria included histopathological evidence of cirrhosis, significant cardiac disease, total splenectomy, and use of agents that are hepatotoxic, promote bleeding, or inhibit olipudase alfa.

\section{Study drug}

For each infusion, lyophilized olipudase alfa was reconstituted in water, diluted in normal saline, and immediately administered intravenously by pump over $20-100 \mathrm{~min}$, according to dose.

\section{Procedures}

Study patients were hospitalized for 3 days, with close monitoring in a cardiac care unit from $6 \mathrm{~h}$ before infusion to $24 \mathrm{~h}$ after infusion and then in the General Clinical Research Center for an additional $48 \mathrm{~h}$. Follow-up assessments were conducted during an overnight visit on day 14 and an outpatient visit on day 28. Liver biopsies were performed at screening and on day 14.

Assessments of olipudase alfa bioactivity in vivo included determination of plasma sphingomyelin, ceramide, and sphingosine concentrations by liquid chromatographytandem mass spectrometry. For single-dose pharmacokinetics, blood samples were obtained before infusion through $72 \mathrm{~h}$ after infusion. Plasma olipudase alfa concentrations were measured by an enzyme-linked immunosorbent assay; values $<156 \mathrm{ng} / \mathrm{ml}$ (lower limit of detection) were excluded from pharmacokinetic analyses, which used noncompartmental methods. Safety was evaluated by AE reporting, telemetry through $24 \mathrm{~h}$ after the dose, and periodic laboratory and vital organ function assessments (Supplementary Table S1 online). Following study completion, frozen stored serum or plasma samples from the eight patients in the four highest dose cohorts were assayed for 89 biomarkers of inflammatory and immune pathways (Human Multi-Analyte Profiling version 1.6; Myriad RBM, Austin, TX).

\section{RESULTS}

\section{Patient population and dose cohorts}

Twenty-three patients were screened, 13 met eligibility criteria, and 11 were infused with olipudase alfa and completed the study (Table 1). Two eligible patients were not treated (lost to followup, study termination for DLT). The two lowest dose cohorts enrolled three patients each. Higher-dose cohorts were to enroll two patients each, but only one patient received the highest dose before study termination. ASM genotype and enzyme activity were consistent with NPD B, and baseline evaluation of hepatosplenomegaly, hematologic parameters, and pulmonary function indicated moderate to severe clinical disease in all patients.

\section{Pharmacokinetics}

The maximum concentration of olipudase alfa occurred at the end of the infusion for each patient. Dose proportionality was observed for the maximum concentration and area under the plasma concentration-time curve (to final concentration $\left(\mathrm{AUC}_{0-t}\right)$ and to infinity $\left(\mathrm{AUC}_{0-\dot{\omega}}\right)$ ), except for patient 8 , who had a high body mass index and received a $0.3 \mathrm{mg} / \mathrm{kg}$ dose 
but exhibited plasma concentrations more consistent with the $0.6 \mathrm{mg} / \mathrm{kg}$ cohort. Dosing was based on total body weight, not lean body mass. For the lowest dose cohort, olipudase alfa was undetectable by $4 \mathrm{~h}$ after the dose. For the other dose cohorts, $t_{1 / 2}$ ranged from 10 to $15 \mathrm{~h}$, and the apparent volume of distribution $\left(V_{z}\right)$ ranged from 9-19l.

\section{Bioactivity}

Plasma concentrations of sphingomyelin and ceramide were within normal limits for all patients at baseline (Table 1 and Figure 1). No postinfusion changes in sphingomyelin concentrations were evident except for unexpected increases within the normal range between 24 and $72 \mathrm{~h}$ in patients $10(0.6 \mathrm{mg} /$ $\mathrm{kg}$ ) and $11(1.0 \mathrm{mg} / \mathrm{kg})$, possibly reflecting cellular damage and the release of stored sphingomyelin. Patient 11 had laboratory evidence of mild liver disease at baseline (discussed below). Ceramide concentrations increased up to 5 -fold in a dose- and time-dependent fashion, beginning between 2 and $6 \mathrm{~h}$ after infusion, peaking between 24 and $48 \mathrm{~h}$, and returning to preinfusion levels by day 14 (Figure 1). Sphingosine, a ceramide derivative, was not detectable in plasma.

\section{AEs and dose-limiting toxicity}

No deaths or drug-related serious AEs occurred. No cardiovascular changes were detected by telemetry, electrocardiography, echocardiography, or cardiac biomarkers, and there was no evidence of adrenal dysfunction. Overall, 35 treatment-emergent AEs were reported in nine patients; 23 were assessed as drugrelated (adverse drug reactions, ADR) (ADRs), and none were reported at olipudase alfa doses of 0.03 or $0.1 \mathrm{mg} / \mathrm{kg}$ (Table 2). For the five patients who received doses $\geq 0.3 \mathrm{mg} / \mathrm{kg}$, no ADRs occurred during the infusions, but beginning at $12 \mathrm{~h}$ after the dose, four of the patients experienced AEs consistent with an acute phase reaction. These patients experienced constitutional symptoms (fever, pain, nausea, or vomiting) and laboratory changes in positive (high-sensitivity C-reactive protein (hsCRP), ferritin, fibrinogen, and percentage of neutrophils) and negative (transferrin, iron, and albumin) acute phase reactants that peaked between 48 and $72 \mathrm{~h}$ following infusion. All AEs were assessed as mild or moderate in intensity except for severe hyperbilirubinemia $(80.4 \mu \mathrm{mol} / \mathrm{l})$ in patient $11(1.0 \mathrm{mg} / \mathrm{kg})$. Most AEs did not require intervention and resolved within 1 or 2 days.

Prospectively-defined criteria for potential DLT were met by six patients (Table 3). Except for one serious AE of hospitalization for procedural pain following the liver biopsy on day 14 (patient 5), the DLT criteria primarily involved increased bilirubin or hsCRP concentrations, as follows.

Patient $2(0.03 \mathrm{mg} / \mathrm{kg})$ had normal bilirubin concentrations throughout the study, but a DLT criterion was met because of a 2 -fold increase in direct (conjugated) bilirubin $24 \mathrm{~h}$ after infusion (total $12.0 \mu \mathrm{mol} / \mathrm{l}$; direct $3.4 \mu \mathrm{mol} / \mathrm{l}$ ). Other liver function tests and biopsy results were unremarkable.

Patient $8(0.3 \mathrm{mg} / \mathrm{kg})$, whose pharmacokinetic profile corresponded to a dose of $0.6 \mathrm{mg} / \mathrm{kg}$ (see above), had a 6 -fold increase in hsCRP and a transient $20 \%$ decrease in platelet count from baseline. The hsCRP concentration peaked at $\sim 4 \times$ the upper limit of normal and was accompanied by a nonclinically significant increase in percentage of neutrophils and a decrease in serum iron. The patient reported mild leg pains, vague abdominal complaints, and nausea between 12 and $24 \mathrm{~h}$ postinfusion, and a low-grade fever $\left(38^{\circ} \mathrm{C}\right)$ peaked at $36 \mathrm{~h}$. Constitutional symptoms resolved by $72 \mathrm{~h}$ and laboratory

Table 1 Patient demographics and baseline characteristics

\begin{tabular}{|c|c|c|c|c|c|c|c|c|c|c|c|c|c|c|}
\hline \multirow{2}{*}{$\begin{array}{l}\text { Dose } \\
\text { (mg/ } \\
\mathrm{kg})\end{array}$} & \multirow[b]{2}{*}{ Patient } & \multirow[b]{2}{*}{ Sex } & \multirow[b]{2}{*}{ Ethnicity } & \multicolumn{2}{|c|}{$\begin{array}{l}\text { SMPD1a gene } \\
\text { mutations }^{\mathrm{b}}\end{array}$} & \multirow{2}{*}{$\begin{array}{c}\text { Age } \\
\text { (years) }\end{array}$} & \multirow{2}{*}{$\begin{array}{l}\text { BMI } \\
(\mathrm{kg} / \\
\left.\mathrm{m}^{2}\right)\end{array}$} & \multirow{2}{*}{$\begin{array}{c}\text { ASM } \\
\text { activity } \\
\text { (\% of } \\
\text { normal in } \\
\text { leukocytes) }\end{array}$} & \multirow{2}{*}{$\begin{array}{c}\text { Plasma } \\
\text { sphin- } \\
\text { gomyelinc } \\
(\mu \mathrm{g} / \mathrm{ml})\end{array}$} & \multirow{2}{*}{$\begin{array}{c}\text { Spleen } \\
\text { volume } \\
\text { (MN) }\end{array}$} & \multirow{2}{*}{$\begin{array}{l}\text { Liver } \\
\text { volume } \\
\text { (MN) }\end{array}$} & \multirow{2}{*}{$\begin{array}{c}\text { Platelet } \\
\text { count } \\
\left(\times 10^{9} / l\right)\end{array}$} & \multirow{2}{*}{$\begin{array}{l}\text { Hemo- } \\
\text { globin } \\
\text { (g/l) }\end{array}$} & \multirow{2}{*}{$\begin{array}{c}\mathrm{DL}_{\mathrm{co}} \\
\text { (\% of } \\
\text { predicted) }\end{array}$} \\
\hline & & & & Allele 1 & Allele 2 & & & & & & & & & \\
\hline \multirow[t]{3}{*}{0.03} & 1 & $\mathrm{M}$ & Caucasian & p.R600H & p.R608del & 21 & 23.3 & 25 & 344 & 15.1 & 2.1 & 186 & 132 & 44 \\
\hline & 2 & M & Caucasian & p.R600H & p.R608del & 19 & 21.0 & 15 & 312 & 16.1 & 2.1 & 186 & 132 & 40 \\
\hline & 3 & $\mathrm{~F}$ & Caucasian & p.L260fs & p.R474W & 23 & 29.3 & 14 & 428 & 12.5 & 1.7 & 148 & 144 & 56 \\
\hline 0.1 & 6 & $\mathrm{~F}$ & Caucasian & p.G242R & p.N383S & 41 & 22.0 & 29 & 312 & 8.7 & 1.2 & 179 & 124 & 81 \\
\hline \multirow[t]{2}{*}{0.3} & 7 & M & Caucasian & p.R441X & p.P323A & 24 & 24.3 & 18 & BQL & $9.5^{d}$ & 1.6 & 144 & 149 & 70 \\
\hline & 8 & $\mathrm{~F}$ & Caucasian & p.R608del & p.R496C & 54 & 40.2 & 24 & BQL & 6.1 & 1.2 & 171 & 126 & 68 \\
\hline \multirow[t]{2}{*}{0.6} & 9 & M & Caucasian & p.V143fs & p.R441X & 18 & 21.8 & 12 & BQL & 8.7 & 1.2 & 126 & 133 & 77 \\
\hline & 10 & M & Caucasian & p.G245S & p.R474W & 18 & 21.7 & 6 & 227 & 10.1 & 1.7 & 116 & 146 & 79 \\
\hline
\end{tabular}

ASM, acid sphingomyelinase; BMI, body mass index; BQL, below the quantifiable level; $\mathrm{DL}_{\mathrm{co}}$, diffusing capacity of the lung using carbon monoxide; $\mathrm{F}$, female; $\mathrm{M}$, male; MN, multiples of normal, assuming spleen volume is $0.2 \%$ of body weight and liver volume is $2.5 \%$ of body weight.

aSMPD1 sphingomyelin phosphodiesterase 1. ' ${ }^{b}$ Mutations are described based on the original SMPD1 complementary DNA reference sequence ${ }^{28}$ and historical Human Genome Variation Society (HGVS) nomenclature. ${ }^{29}$ The original complementary DNA sequence is six nucleotides shorter than the current HGVS reference sequence (NM_000543.4) because of the presence of an N-terminal polymorphic signal peptide domain containing five instead of six Ala-Leu repeats. Consequently, the current HGVS reference protein sequence (NP_000534.3) for the longest isoform contains 631 amino acids, with an additional Ala-Leu at positions $48-49$, followed by amino acids whose numbering is increased by two compared with the original protein sequence (i.e., p.R610del versus p. R608del). ${ }^{~} \mathrm{Normal}$ plasma sphingomyelin is $<800 \mathrm{Mg} / \mathrm{ml}$; $\mathrm{BQL}$ is below the quantifiable level of $200 \mu \mathrm{g} / \mathrm{ml}$. ${ }^{\text {PPatient }} 7 \mathrm{had}$ a partial splenectomy 


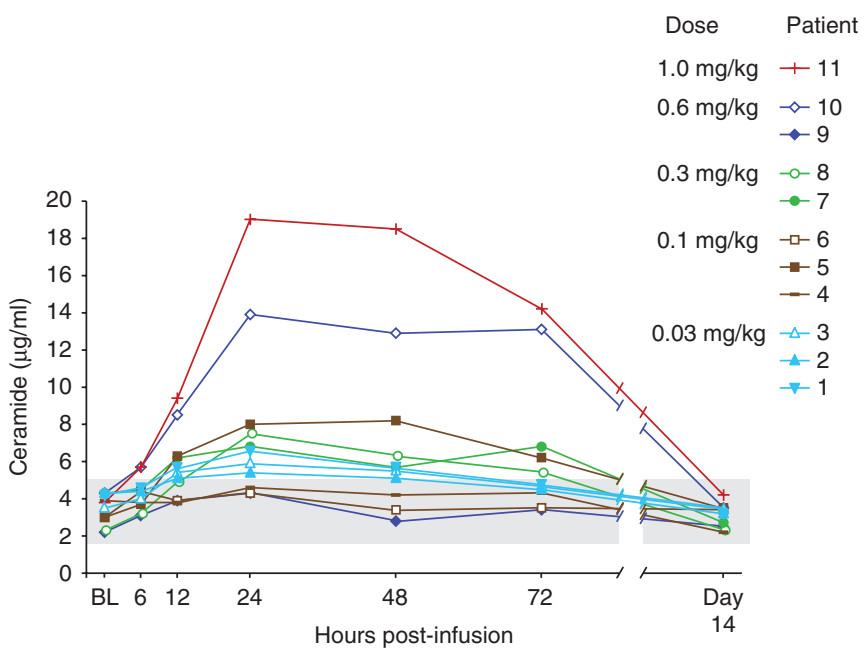

Figure 1 Time course of plasma ceramide concentrations following infusion of olipudase alfa. The shaded area represents the reference range of normal values.

parameters normalized by day 14 . This was the first of four patients to exhibit evidence of an acute phase reaction.

Patient $9(0.6 \mathrm{mg} / \mathrm{kg})$ exhibited a 28 -fold increase $(\sim 8 \times$ the upper limit of normal) in hsCRP during the $72 \mathrm{~h}$ postinfusion. Between 12 and $36 \mathrm{~h}$ postinfusion, the patient reported vague abdominal complaints, headache, and leg pains, which dissipated by day 14 .

Patient $10(0.6 \mathrm{mg} / \mathrm{kg})$ was evaluated for DLT based on laboratory and liver biopsy results. Within $24 \mathrm{~h}$ of dosing, concentrations of bilirubin, hsCRP, and ferritin increased and transferrin decreased. Mild back pain and headache were reported at $48 \mathrm{~h}$. Two small inflammatory foci of lymphocytes, one of which was associated with hepatocyte degeneration, were noted on the liver biopsy on day 14 .

Patient $11(1.0 \mathrm{mg} / \mathrm{kg})$ experienced severe hyperbilirubinemia. The patient had a mildly elevated total bilirubin concentration at baseline $(22.2 \mu \mathrm{mol} / \mathrm{l})$, which increased $\sim 4$-fold during the $48 \mathrm{~h}$ postinfusion and was mostly indirect (total $80.4 \mu \mathrm{mol} / \mathrm{l}$ and direct $18.8 \mu \mathrm{mol} / \mathrm{l}$; normal ranges: total $1.7-$ 20.5 and direct $0.0-13.7)$. At $72 \mathrm{~h}$, urobilinogen was detected and trace scleral icterus noted. There were no elevations in serum ALT, AST, or alkaline phosphatase, and a nonsignificant increase in $\gamma$-glutamyl transpeptidase was noted between 36 and $72 \mathrm{~h}$. Hemoglobin concentrations and hematocrit were stable, although mild reticulocytosis was present. Results from the liver biopsy on day 14 were unchanged from baseline. During the $48 \mathrm{~h}$ postinfusion, evidence of an acute phase reaction included increases in hsCRP, D-dimer, ferritin, fibrinogen, prothrombin time, partial thromboplastin time, and lactate dehydrogenase and decreases in albumin, iron, and transferrin. Beginning $12 \mathrm{~h}$ postinfusion, nausea, vomiting, hypertension, fatigue, and fever were reported. Based on the level of hyperbilirubinemia, the sponsor terminated the study for DLT, and $0.6 \mathrm{mg} / \mathrm{kg}$ was designated as the maximum tolerated starting dose of olipudase alfa in adults with NPD B.

\section{Biomarker analyses}

In the four patients with acute phase reactions, plasma concentrations of ceramide began to rise before hsCRP or bilirubin increased (Figure 2). Inexplicably, the seven prospectively defined cytokines measured during the course of the study were low at all time points when first assayed. Because of possible assay insensitivity or elevations in other cytokines, archived samples were analyzed for a panel of 89 biomarkers using a quantitative immunoassay (see Materials and Methods). Substantial elevations in inflammatory mediators were detected only in the four patients with acute phase reactions. No changes were noted during the $6 \mathrm{~h}$ postinfusion, but between 24 and $72 \mathrm{~h}$, moderate dose-dependent increases in IL-8, calcitonin, and hsCRP occurred (median-fold increases of 26, 23.7, and 21.8 over baseline, respectively), accompanied by small increases (approximately 2-5-fold) in tumor necrosis factor- $\alpha$, macrophage inhibitory protein-1 $\beta$, IL-6, ceramide, tumor necrosis factor-receptor 2 , and bilirubin (Supplementary Table S2 online) and slight increases (less than twofold) in macrophage inflammatory protein-1 $\alpha$, IL-1 $\alpha$, granulocyte colony-stimulating factor, granulocyte-macrophage colony-stimulating factor, and IL- $1 \beta$ (data not shown). The specific biomarkers that increased varied from patient to patient, but all normalized by day 14 . No IgG antibodies to olipudase alfa were detected at baseline or day 28 .

\section{DISCUSSION}

This phase I trial revealed novel dose-dependent, transient ADRs upon first exposure to olipudase alfa in patients with NPD B; these ADRs had the characteristics of acute phase reactions. No ADRs occurred during or immediately after enzyme infusions, but constitutional symptoms began to emerge approximately $12 \mathrm{~h}$ postinfusion, along with elevations in acute phase reactants and bilirubin that peaked between 48 and $72 \mathrm{~h}$ postinfusion. The nature and timing of the firstdose ADRs observed with olipudase alfa are distinct from the usual hypersensitivity-type IARs arising after several doses of ERT in some patients with other lysosomal storage disorders. In most patients with hypersensitivity-type IARs, the detection of antibodies to the enzyme indicates the induction of an adaptive humoral immune response that may account for the adverse effects (although some patients with IARs do not have detectable antibodies and vice versa)., ${ }^{2,3}$ Because each patient received only a single infusion in this phase I trial, no specific antibody formation was expected and none was detected. Instead, the pattern of positive and negative acute phase proteins and cytokines that peaked in the circulation 2-3 days after infusion is consistent with changes in hepatic protein synthesis related to an acute inflammatory response by the innate immune system. . $^{16,17}$

Acute phase reactions have been reported after bisphosphonate administration and are characterized by flu-like symptoms that have been attributed to increased levels of tumor necrosis factor- $\alpha$ and IL- 6 in the circulation resulting from indirect activation of $\gamma \delta \mathrm{T}$ cells. ${ }^{17}$ For olipudase alfa, a possible initiator of 
Table 2 Treatment-emergent adverse events

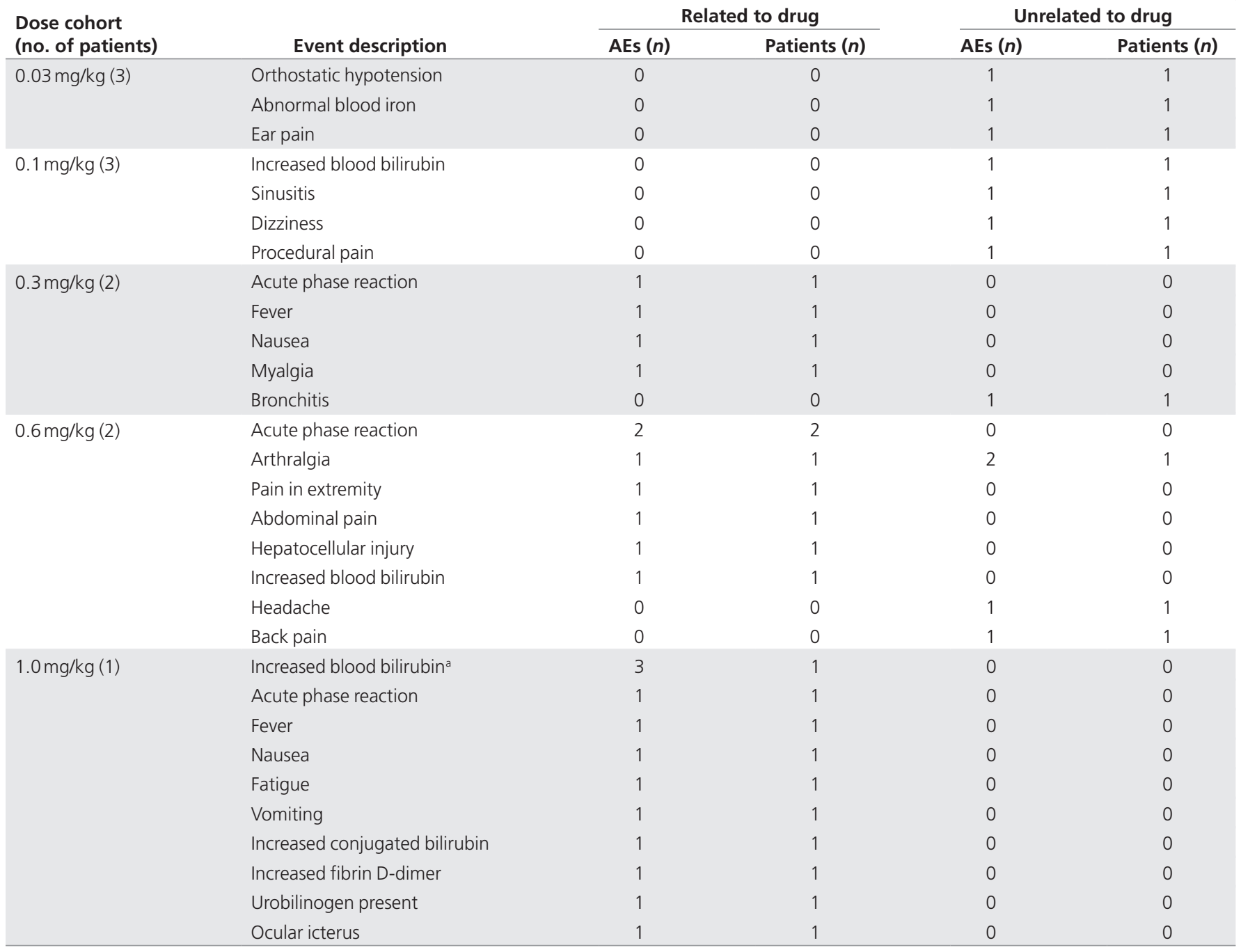

$\mathrm{AE}$, adverse event.

ancreased bilirubin concentrations were reported for patient 11 on days 1,2 , and 3 .

Table 3 Occurrences of potential dose-limiting toxicity evaluated by the data monitoring committee

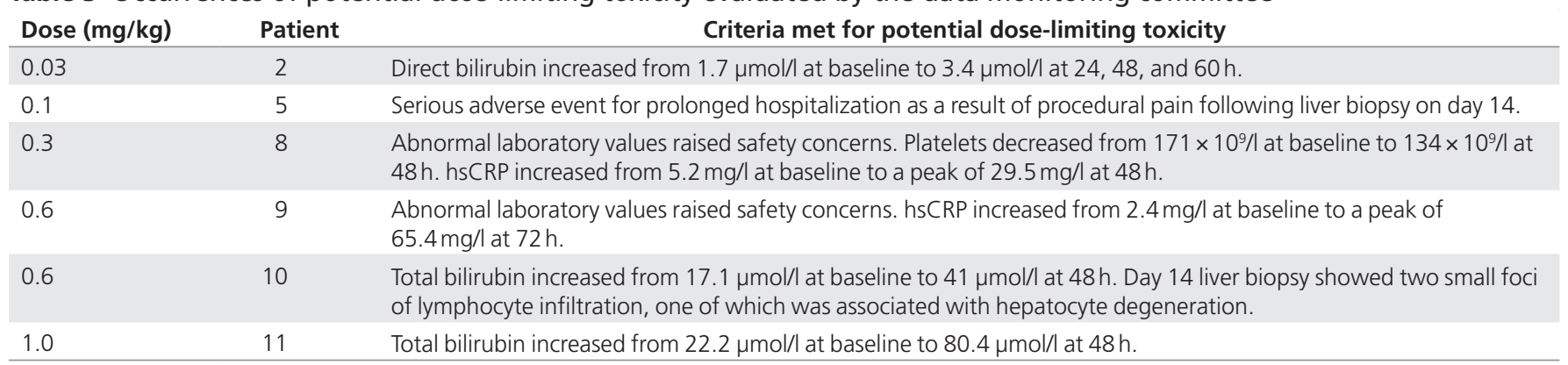

hsCRP, high-sensitivity C-reactive protein.

the acute phase reaction is ceramide, a well-known signaling molecule in pathways that regulate cytokine release, inflammation, and apoptosis. ${ }^{18,19}$ Increasing concentrations of plasma ceramide paralleled the onset of constitutional symptoms and preceded changes in concentrations of bilirubin and acute phase reactants. Although one patient with an acute phase reaction did not exhibit an increase in plasma ceramide, significant correlations between the peak concentrations of ceramide and peak concentrations of hsCRP $(r=0.68 ; P=0.02)$ and total bilirubin ( $r=0.95 ; P=0.001)$ were found. The involvement of 

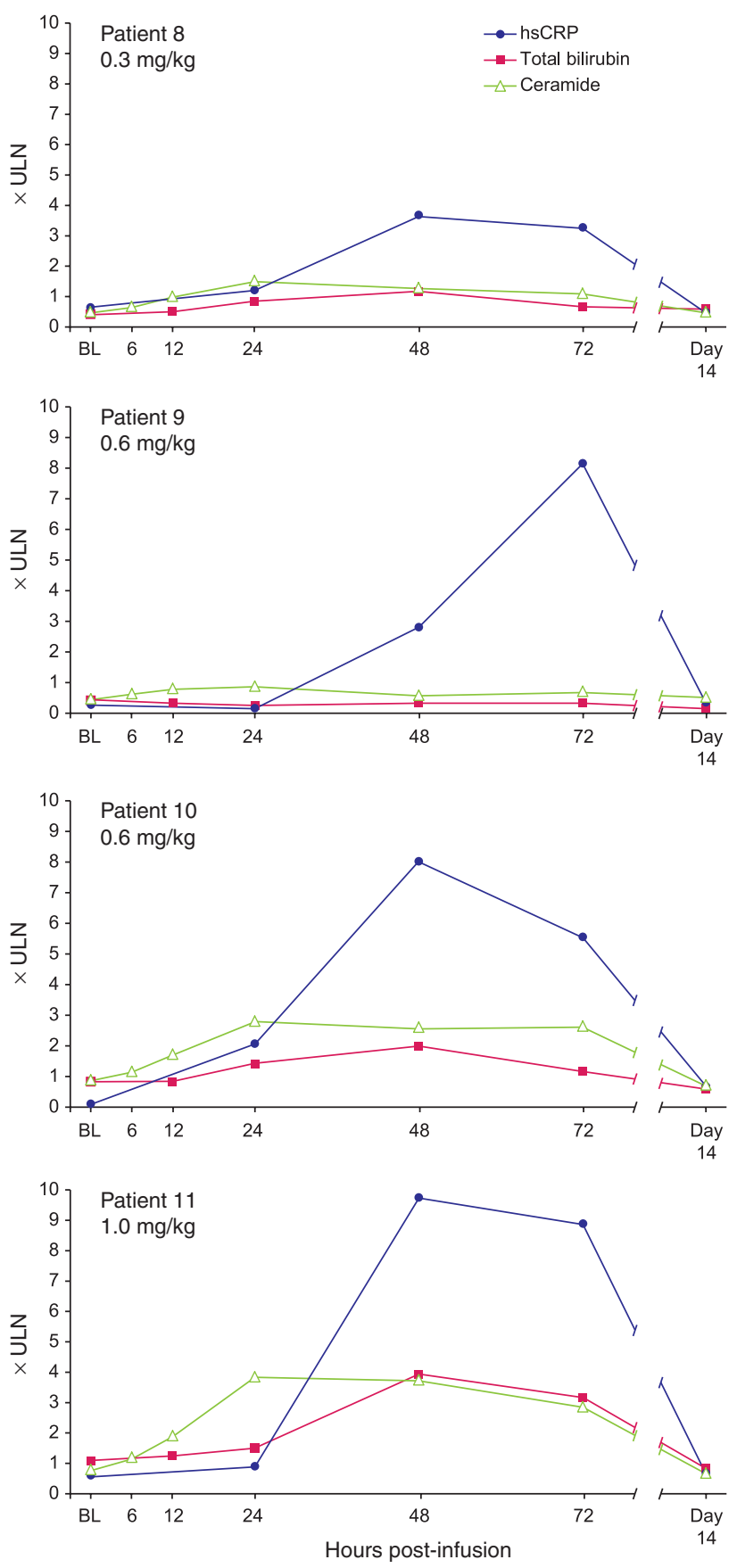

Figure 2 Time course of plasma high-sensitivity C-reactive protein (hsCRP), total bilirubin, and ceramide concentrations in the four patients with acute phase reactions following olipudase alfa infusion. Concentrations are expressed as the upper limit of normal (ULN).

phosphorylcholine (the other ASM end product) or bioactive lipids downstream from ceramide cannot be discounted.

When considering the possibility that the first-dose ADRs to olipudase alfa are initiated directly or indirectly by its end product(s), it is relevant that its substrate, sphingomyelin, is a major component of plasma membranes and circulating lipoprotein particles. Despite plasma sphingomyelin concentrations being normal in patients, intravascular sources of sphingomyelin could still contribute to the increase in plasma ceramide that occurred within hours of the infusion because olipudase alfa retains a low level of activity at a neutral $\mathrm{pH}$ (Genzyme data on file) and has an unusually long circulating $t_{1 / 2}$ for an ERT (10$15 \mathrm{~h}$ vs. $<10 \mathrm{~min}$ to $4 \mathrm{~h}$ for other ERTs). The slow clearance of olipudase alfa from the circulation may be a result of its binding to lipoprotein particles or cell membranes ${ }^{20}$ and/or impaired targetcell uptake via mannose-6-phosphate receptors. ${ }^{21}$ Interestingly, the types of ADRs observed upon first exposure to olipudase alfa have not been reported in patients with Gaucher disease receiving imiglucerase ERT, even though ceramide is a catabolite of glucosylceramide, the enzyme substrate. The rapid cellular uptake of imiglucerase from plasma $\left(t_{1 / 2}<10 \mathrm{~min}\right)$, coupled with the 100 fold lower plasma concentration of glucosylceramide relative to sphingomyelin, may limit the amount of ceramide formed in the circulation in patients with Gaucher disease.

The hyperbilirubinemia that occurred in three of the four patients with an acute phase reaction was mostly indirect and not associated with hemolysis (stable hemoglobin and hematocrit), transaminitis (stable ALT and AST), or cholestasis (stable alkaline phosphatase and $\gamma$-glutamyl transpeptidase). Liver biopsies did not reveal hepatocyte damage, except possibly in one patient, but early olipudase alfa-induced pathology may have resolved by day 14 when postinfusion tissue was obtained. In patients with NPD B, sphingomyelin accumulates in hepatocytes as well as in Kupffer cell macrophages. ${ }^{22}$ Increased intracellular ceramide concentrations generated by olipudase alfa might limit the availability of UDP-glucuronide for bilirubin conjugation by sequestering UDP as UDP-galactose and UDP-glucose for the conversion of ceramide to galactosylceramide and glucosylceramide, respectively. Direct effects of ceramide on lipid bilayers might also impair bilirubin uptake by organic anion transporters within the basolateral membrane and/or inhibit the activity of UDP-glucuronyltransferase within the endoplasmic reticulum of hepatocytes. ${ }^{19,23,24}$ In this regard, patient 11 , who had a slightly elevated bilirubin concentration at baseline and developed severe hyperbilirubinemia after infusion, was found retrospectively to be homozygous for the common (TA)7 promoter mutation that causes Gilbert syndrome, an autosomal-recessive condition associated with $\sim 30 \%$ of normal UDPglucuronyltransferase activity. ${ }^{25}$ This patient experienced an increase in cortisol, indicative of a physiologic stress response, a known trigger for hyperbilirubinemia in patients with Gilbert syndrome. The two other patients with mild hyperbilirubinemia were heterozygous for sequence variants that may predispose to Gilbert syndrome. Gilbert syndrome is a common disorder that is found in up to $10 \%$ of the population. ${ }^{26}$ Indirect hyperbilirubinemia also has been reported as an ADR to the antiretroviral protease inhibitors atazanavir and indinavir. ${ }^{27}$ These drugs directly inhibit bilirubin UDP-glucuronyltransferase activity, making individuals with Gilbert syndrome more susceptible to developing hyperbilirubinemia and jaundice.

This phase I study identified $0.6 \mathrm{mg} / \mathrm{kg}$ as the maximum tolerated starting dose of olipudase alfa in patients with NPD 
B. The results of this and previous preclinical studies support the use of a within-patient dose-escalation strategy, using low doses of olipudase alfa to gradually clear accumulated sphingomyelin before escalating to higher doses. Importantly, the study also identified potential biomarkers, including ceramide, bilirubin, hsCRP, and other acute phase reactants, that should prove useful for monitoring the safety of repeated olipudase alfa infusions in patients with ASMD.

\section{SUPPLEMENTARY MATERIAL}

Supplementary material is linked to the online version of the paper at http://www.nature.com/gim

\section{ACKNOWLEDGMENTS}

The study was sponsored by Genzyme Corporation and supported by grants to M.M.M. (NIH K24) and grant UL1TR000067 from the National Center for Advancing Translational Sciences (NCATS), a component of the National Institutes of Health (NIH). Its contents are solely the responsibility of the authors and do not necessarily represent the official views of NCATS or the NIH. Andrea M. Norfleet (Genzyme) provided writing assistance, Fanny O'Brien (Genzyme) conducted statistical analyses, and William Kramer Consulting performed pharmacokinetic analyses.

\section{DISCLOSURE}

The study was sponsored by Genzyme Corporation, which collaborated with the investigators on the study design, interpretation of results, preparation of the manuscript, and decision to submit the manuscript for publication. M.M.M. was the principal investigator for the study. M.P.W. has consulted for, and received research support from, Genzyme, a Sanofi company. B.L.T., S.R., and G.F.C. are employees of Genzyme, a Sanofi company. The other authors declare no conflict of interest.

\section{REFERENCES}

1. Hollak CE, Wijburg FA. Treatment of lysosomal storage disorders: successes and challenges. J Inherit Metab Dis 2014;37:587-598.

2. Richards SM. Immunologic considerations for enzyme replacement therapy in the treatment of lysosomal storage disorders. Clin Appl Immunol Rev 2002;2:241-253.

3. Starzyk K, Richards S, Yee J, Smith SE, Kingma W. The long-term international safety experience of imiglucerase therapy for Gaucher disease. Mol Genet Metab 2007;90:157-163.

4. Schuchman EH, Desnick RJ. Niemann-Pick disease types A and B: acid sphingomyelinase deficiencies. In: Valle D, Beaudet AL, Vogelstein B, et al (eds). OMMBID - The Online Metabolic and Molecular Bases of Inherited Diseases. McGraw-Hill: New York, 2013. http://ommbid.mhmedical. com/content. aspx? bookid=474\&Sectionid $=45374145$. Accessed 24 September 2014

5. McGovern MM, Lippa N, Bagiella E, Schuchman EH, Desnick RJ, Wasserstein MP. Morbidity and mortality in type B Niemann-Pick disease. Genet Med 2013:15:618-623.

6. McGovern MM, Aron A, Brodie SE, Desnick RJ, Wasserstein MP. Natural history of Type A Niemann-Pick disease: possible endpoints for therapeutic trials. Neurology 2006;66:228-232.

7. McGovern MM, Wasserstein MP, Giugliani R, et al. A prospective, crosssectional survey study of the natural history of Niemann-Pick disease type B. Pediatrics 2008; 122:e341-e349.

8. Wasserstein MP, Desnick RJ, Schuchman EH, et al. The natural history of type B Niemann-Pick disease: results from a 10-year longitudinal study. Pediatrics 2004;114:e672-e677.
9. Mylla Neto G, Costa R, Fernandes PM, de Lima EC, Ribeiro FM, Stolf NA. Niemann-Pick disease in adult: report of a case surgically treated. Rev Hosp Clin Fac Med Sao Paulo 1983;38:83-85.

10. Nicholson AG, Wells AU, Hooper J, Hansell DM, Kelleher A, Morgan C. Successful treatment of endogenous lipoid pneumonia due to NiemannPick Type B disease with whole-lung lavage. Am J Respir Crit Care Med 2002;165:128-131.

11. Daloze P, Delvin EE, Glorieux FH, Corman JL, Bettez P, Toussi T. Replacement therapy for inherited enzyme deficiency: liver orthotopic transplantation in Niemann-Pick disease type A. Am J Med Genet 1977;1:229-239.

12. Mendes MS, Portela FX, Reis RC, Castro JD, Garcia JH, Holanda MA. Liver transplantation in a patient with Niemann-Pick disease and pulmonary involvement. J Bras Pneumol 2012;38:269-271

13. Shah AJ, Kapoor N, Crooks GM, et al. Successful hematopoietic stem cell transplantation for Niemann-Pick disease type B. Pediatrics 2005;116:1022-1025.

14. Miranda SR, He X, Simonaro CM, et al. Infusion of recombinant human acid sphingomyelinase into niemann-pick disease mice leads to visceral, but not neurological, correction of the pathophysiology. FASEB J 2000;14:1988-1995.

15. Murray JM, Thompson AM, Vitsky A, et al. Nonclinical safety assessment of recombinant human acid sphingomyelinase (rhASM) for the treatment of acid sphingomyelinase deficiency: The utility of animal models of disease in the toxicological evaluation of potential therapeutics. Mol Genet Metab 2015:114:217-225.

16. Colley CM, Fleck A, Goode AW, Muller BR, Myers MA. Early time course of the acute phase protein response in man. J Clin Pathol 1983;36:203-207.

17. Reid IR, Gamble GD, Mesenbrink P, Lakatos P, Black DM. Characterization of and risk factors for the acute-phase response after zoledronic acid. J Clin Endocrinol Metab 2010;95:4380-4387.

18. Spiegel S, Foster D, Kolesnick R. Signal transduction through lipid second messengers. Curr Opin Cell Biol 1996;8:159-167.

19. Gulbins E, Dreschers S, Wilker B, Grassmé H. Ceramide, membrane rafts and infections. J Mol Med (Berl) 2004;82:357-363.

20. Schissel SL, Jiang X, Tweedie-Hardman J, et al. Secretory sphingomyelinase, a product of the acid sphingomyelinase gene, can hydrolyze atherogenic lipoproteins at neutral pH. Implications for atherosclerotic lesion development. J Biol Chem 1998:273:2738-2746.

21. Dhami R, Schuchman EH. Mannose 6-phosphate receptor-mediated uptake is defective in acid sphingomyelinase-deficient macrophages: implications for Niemann-Pick disease enzyme replacement therapy. J Biol Chem 2004:279:1526-1532.

22. Thurberg BL, Wasserstein MP, Schiano T, et al. Liver and skin histopathology in adults with acid sphingomyelinase deficiency (Niemann-Pick disease type B). Am J Surg Pathol 2012;36:1234-1246.

23. Cui Y, König J, Leier I, Buchholz U, Keppler D. Hepatic uptake of bilirubin and its conjugates by the human organic anion transporter SLC21A6. J Biol Chem 2001;276:9626-9630.

24. Hauser SC, Ziurys JC, Gollan JL. Subcellular distribution and regulation of hepatic bilirubin UDP-glucuronyltransferase. J Biol Chem 1984;259:4527-4533.

25. Cappellini MD, Martinez di Montemuros F, Sampietro M, Tavazzi D, Fiorelli G. The interaction between Gilbert's syndrome and G6PD deficiency influences bilirubin levels. Br J Haematol 1999;104:928-929.

26. Strassburg CP. Gilbert-Meulengracht's syndrome and pharmacogenetics: is jaundice just the tip of the iceberg? Drug Metab Rev 2010;42:168-181.

27. Zucker SD, Qin X, Rouster SD, et al. Mechanism of indinavir-induced hyperbilirubinemia. Proc Natl Acad Sci USA 2001;98:12671-12676.

28. Schuchman EH, Suchi M, Takahashi T, Sandhoff K, Desnick RJ. Human acid sphingomyelinase. Isolation, nucleotide sequence and expression of the fulllength and alternatively spliced CDNAs. J Biol Chem 1991;266:8531-8539.

29. Antonarakis SE. Recommendations for a nomenclature system for human gene mutations. Nomenclature Working Group. Hum Mutat 1998;11:1-3.

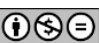

This work is licensed under a Creative Commons Attribution-NonCommercial-NoDerivs 4.0 International License. The images or other third party material in this article are included in the article's Creative Commons license, unless indicated otherwise in the credit line; if the material is not included under the Creative Commons license, users will need to obtain permission from the license holder to reproduce the material. To view a copy of this license, visit http://creativecommons.org/licenses/by-nc-nd/4.0/ 